\title{
Phytoprotection
}

\section{Inventaire des mauvaises herbes dans les pépinières ornementales du Québec}

\author{
D.C. Cloutier, M.L. Leblanc et R. Marcotte
}

Volume 72, numéro 2, 1991

URI : https://id.erudit.org/iderudit/706002ar

DOI : https://doi.org/10.7202/706002ar

Aller au sommaire du numéro

Éditeur(s)

Société de protection des plantes du Québec (SPPQ)l

ISSN

0031-9511 (imprimé)

1710-1603 (numérique)

Découvrir la revue

Citer cet article

Cloutier, D., Leblanc, M. \& Marcotte, R. (1991). Inventaire des mauvaises herbes dans les pépinières ornementales du Québec. Phytoprotection, 72(2), 41-51. https://doi.org/10.7202/706002ar
Résumé de l'article

Un inventaire des mauvaises herbes réalisé au cours de l'été 1986 a permis de visiter 53\% des superficies en production ornementale au Québec. Les infestations de mauvaises herbes étaient maintenues à de faibles niveaux dans les pépinières visitées grâce aux sarclages manuels et aux hersages mécaniques fréquents. L'inventaire a révélé que les mauvaises herbes vivaces étaient les plus difficiles à réprimer dans les pépinières. Les mauvaises herbes vivaces les plus fréquemment rencontrées étaient la vesce jargeau (Vicia cracca), le chiendent (Agropyron repens), la prèle des champs (Equisetum arvense) et le souchet comestible (Cyperus esculentus). Les mauvaises herbes identifiées dans les pépinières étaient, pour la plupart, des espèces communes à d'autres cultures. Toutefois, la rorippe d'Islande (Rorippa islandica), la rorippe sylvestre (Rorippa sylvestris) et la cardamine de Pennsylvanie (Cardamine pensylvanica), mauvaises herbes moins connues, ont démontré un fort potentiel d'envahissement dans les pépinières ornementales. 


\title{
Inventaire des mauvaises herbes dans les pépinières ornementales du Québec
}

\author{
D. C. Cloutier, M. L. Leblanc et R. Marcotte \\ Ferme expérimentale, Agriculture Canada, C.P. 3398, L'Assomption (Québec), \\ Canada JOK 1GO. Contribution No 335/91.03.01R
}

(Reçu 1990-09-14; accepté 1991-04-25)

\begin{abstract}
Un inventaire des mauvaises herbes réalisé au cours de l'été 1986 a permis de visiter 53\% des superficies en production ornementale au Québec. Les infestations de mauvaises herbes étaient maintenues à de faibles niveaux dans les pépinières visitées grâce aux sarclages manuels et aux hersages mécaniques fréquents. L'inventaire a révélé que les mauvaises herbes vivaces étaient les plus difficiles à réprimer dans les pépinières. Les mauvaises herbes vivaces les plus fréquemment rencontrées étaient la vesce jargeau (Vicia cracca), le chiendent (Agropyron repens), la prèle deschamps (Equisetumarvense) et le souchet comestible (Cyperus esculentus). Les mauvaises herbes identifiées dans les pépinières étaient, pour la plupart, des espèces communes à d'autres cultures. Toutefois, la rorippe d'Islande (Rorippa islandica), la rorippe sylvestre (Rorippa sylvestris) et la cardamine de Pennsylvanie (Cardamine pensylvanica), mauvaises herbes moins connues, ont démontré un fort potentiel d'envahissement dans les pépinières ornementales.
\end{abstract}

Cloutier, D.C., M.L. Leblanc et R. Marcotte. 1991. Inventaire des mauvaises herbes dans les pépinières ornementales du Québec. PHYTOPROTECTION 72: 41-51.

A weed survey of fields in ornamental production was conducted in Quebec in 1986 during which $53 \%$ of the production sites were visited. Because of the intensity of hand-weeding and mechanical cultivation used in the fields, no major weed infestations were observed. Weeds most difficult to control were mainly perennial species such as tufted vetch (Vicia cracca), quackgrass (Agropyron repens), field horsetail (Equisetum arvense), and yellow nutsedge (Cyperus esculentus). Most of the weeds identified were species common to other crops. However, marsh yellow cress (Rorippa islandica), yellow field cress (Rorippa sylvestris), and bitter cress (Cardamine pensylvanica) were lesser known weed species that showed a great potential of increase in ornamental nursery production.

\section{Introduction}

L'horticulture ornementale au Québec est une industrie qui a une production annuelle d'une valeur de 15 à 30 millions de dollars (Statistique Canada 1990). Elle regroupe 300 producteurs qui cultivent un millier d'hectares (MAPAQ 1987) (tableau 1). Les mauvaises herbes qui infestent les grandes cultures et les cultures maraîchères ont été répertoriées au cours des années 1979-1982 au Québec (Doyon et al. 1982). Cependant, il n'y a aucune information disponible sur la fréquence et l'abondance des mauvaises herbes dans la production ornementale et sur l'importance des problèmes qu'elles occasionnent. En Europe et aux ÉtatsUnis, les pépiniéristes considèrent les mauvaises herbes comme étant le principal facteur limitatif de production (Robinson 1983; Whitcomb 1984). Par conséquent, il devenait donc critique d'établir un état de la situation dans les pépinières afin d'identifier les besoins de recherches en malherbologie dans ce secteur de production. Au cours de l'été 1986, une enquête a été réalisée afin d'inventorier les espèces de mauvaises herbes présentes dans les pépinières du Québec. Les objectifs spécifiques de l'enquête étaient de déterminer la fréquence de chaque espèce et de recueillir les données concernant les pratiques culturales et les méthodes de répression utilisées par les pépiniéristes. La production ornementale en pleine terre et en contenants ont toutes deux été incluses dans l'inventaire.

\section{Matériel et méthodes}

Les pépinières visitées ont été sélectionnées à partir de la liste des pépinières inspectées et approuvées par le Service des productions végétales du Ministère de l'Agriculture, des Pêcheries et de l'Alimentation du Québec (MAPAQ 1985). Seules les pépinières de 3 ha et plus ont été considérées. Des 26 pépiniéristes consultés, 22 ont accepté de participer à l'inventaire. Ils étaient répartis dans les régions de Québec, du Nord de Montréal, du Richelieu, du Sud-Ouest de Montréal et de la Mauricie(tableau 1). Onze pépiniéristes produisaient en pleine terre et en contenants, 10 autres produisaient exclusivement en pleine terre alors qu'un pépiniériste produisait exclusivement en contenants (tableau 1). Les pépinières ont été visitées une fois entre le 24 juillet et le 5 septembre 1986. La visite des pépinières consistait à inspecter systématiquement les sites de production avec le 
Tableau 1. Superficie totale des pépinières de plantes ornementales en production selon les régions au Québec en 1986 et superficie relative des pépinières visitées au cours de l'enquête

\begin{tabular}{|c|c|c|c|c|c|c|}
\hline $\begin{array}{l}\text { Numéro } \\
\text { des } \\
\text { régions }\end{array}$ & $\begin{array}{l}\text { Nom des } \\
\text { régions }\end{array}$ & $\begin{array}{l}\text { Nombre de } \\
\text { pépinières } \\
\text { de la } \\
\text { région }\end{array}$ & $\begin{array}{l}\text { Superficie } \\
\text { de toutes les } \\
\text { pépinières } \\
\text { de la région } \\
\text { (ha) }\end{array}$ & $\begin{array}{l}\text { Superficie des } \\
\text { pépinières } \\
\text { visitées } \\
\text { (\% de la région) }\end{array}$ & $\begin{array}{c}\text { Nombre total } \\
\text { de pépinières } \\
\text { visitées }\end{array}$ & $\begin{array}{l}\text { Nombre de } \\
\text { pépinières } \\
\text { visitées } \\
\text { produisant en } \\
\text { contenants }\end{array}$ \\
\hline 1 & Bas Saint-Laurent / Gaspésie & 11 & 3,4 & & & \\
\hline 2 & Québec & 39 & 80,7 & 64 & 5 & $5^{\dagger}$ \\
\hline 3 & Beauce & 14 & 9,2 & & & \\
\hline 4 & Nicolet & 20 & 31,2 & & & \\
\hline 5 & Estrie & 13 & 20,0 & & & \\
\hline 6 & Richelieu & 43 & 274,3 & 44 & 5 & 3 \\
\hline 7 & Sud-Ouest de Montréal & 34 & 280,5 & 84 & 1 & 0 \\
\hline 8 & Outaouais & 9 & 11,8 & & & \\
\hline 9 & Nord-Ouest Québécois & 10 & 7,4 & & & \\
\hline 10 & Nord de Montréal & 87 & 200,9 & 38 & 10 & 4 \\
\hline 11 & Mauricie & 13 & 37,1 & 70 & 1 & () \\
\hline \multirow[t]{2}{*}{12} & Saguenay / Lac Saint-Jean & 11 & 4,5 & & & \\
\hline & Ensemble du Québec & 304 & 961,0 & 53 & 22 & 12 \\
\hline
\end{tabular}

$\S$ MAPAQ 1987.

† Une pépinière produisait exclusivement en contenants.

producteur ou une personne-ressource permettant ainsi d'obtenir des renseignements additionnels relatifs à la régie. Tous les champs en production étaient parcourus sur toute leur superficie en suivant un trajet en zigzag de long en large. Dans chacune des pépinières, toutes les mauvaises herbes retrouvées étaient identifiées et le type de programme de répression des mauvaises herbes était aussi noté. Le recouvrement et l'abondance des espèces n'étaient pas notés parce que ceux-ci étaient généralement inférieurs à $1 \%$. Un échantillon représentatif $\left(0,015 \mathrm{~m}^{3}\right)$ du terreau utilisé pour la production en contenants a été prélevé chez chacun des sept pépiniéristes qui en avaient en réserve. L'échantillon était conservé dans des sacs de plastique pour une période ne dépassant pas 24 het par la suite, il était séché à l'air ambiant pour préserver les graines.

Chaque échantillon a été déposé dans trois plateaux de $28 \mathrm{~cm}$ x $53 \mathrm{~cm}$ x $6 \mathrm{~cm}$, mis en serre le 2 décembre 1986 et observé pendant 12 mois. Le terreau était maintenu humide et mélangé tous les 2 mois pour stimuler la germination des graines pouvant être présentes dans celui-ci. La serre était maintenue à $25^{\circ} \mathrm{C}$ pendant $8 \mathrm{~h}$ sous éclairage naturel ou artificiel, et à $18^{\circ} \mathrm{C}$ la nuit (l'automne et l'hiver). Au printemps et à l'été, la température était en moyenne de $30^{\circ} \mathrm{C}$ pen- dant la journée (aucune lumière supplémentaire) et de $20^{\circ} \mathrm{C}$ pendant la nuit. Les plantules émergeant du terreau ont été dénombrées, transplantées en pots après le stade cotylédon et gardées jusqu'à ce qu'elles soient identifiées. La nomenclature scientifique des mauvaises herbes est celle de Scoggan (1978-1979) et les noms communs sont ceux d'Alex et al. (1980).

\section{Résultats et discussion}

Les 22 pépinières visitées représentaient $53 \%$ de la surface occupée par les pépinières du Québec en 1986. Les résultats des inventaires de la production en pleine terre sont présentés par région alors que les résultats de l'inventaire de la production en contenants regroupent l'ensemble des régions. Les méthodes de répression sont présentées pour chaque type de production.

Inventaire des mauvaises herbes dans la production en pleine terre. Les données sur les mauvaises herbes dans la production en pleine terre sont regroupées selon les régions: Québec et Mauricie, Richelieu, Sud-Ouest et Nord de Montréal. Les pépinières visitées dans les régions de Québec et de la Mauricie étaient caractérisées par des sols sableux, limoneux ou argileux. Ensemble, elles représentaient respectivement $64 \%$ et $70 \%$ de la surface occupée 
par les pépinières de plantes ornementales de ces régions et $8,1 \%$ de l'étendue totale en pépinière au Québec (tableau 1). La liste des espèces de mauvaises herbes rencontrées est présentée au tableau 2. Les espèces les plus communes étaient la vesce jargeau (Vicia cracca L.), le chiendent (Agropyron repens [L.] Beauv.), la matricaire odorante (Matricaria matricarioides [Less.] Porter) et la rorippe d'Islande ( $R O-$ rippa islandica [Oeder] Borb.).

Les cinq pépinières visitées dans la région du Richelieu étaient établies sur des sols sableux ou limoneux. Chacune d'elles couvrait une superficie de 14 ha et plus. Ensemble, elles représentaient $44 \%$ de la surface occupée par les pépinières de plantes ornementales de cette région et $12,6 \%$ de la superficie totale en pépinière au Québec (tableau 1). Les espèces les plus fréquentes étaient l'amarante à racine rouge (Amaranthus retroflexus L.), le chénopode (Chenopodium spp.), la digitaire sanguine (Digitaria sanguinalis [L.] Scop.), l'échinochloa pied-de-coq (Echinochloa crusgalli [L.] Beauv.), la petite herbe à poux (Ambrosia artemisiifolia L.), le panic capillaire (Panicum capillare L.), la renouée persicaire (Polygonum persicaria $\mathrm{L}$.), la rorippe d'Islande et la stellaire moyenne (Stellaria media [L.] Vill.) (tableau 2).

Les 11 pépinières des régions du Sud-Ouest et du Nord de Montréal étaient caractérisées par des sols sableux, limoneux ou argileux; ensemble, elles représentaient respectivement $84 \%$ et $38 \%$ de la surface occupée par les pépinières de plantes ornementales. Celles-ci constituaient $32,5 \%$ de la superficie totale en pépinière au Québec (tableau 1). Les mauvaises herbes les plus communes étaient le chiendent, le chénopode, l'échinochloa pied-de-coq, la vesce jargeau, l'amarante à racine rouge, la renouée persicaire, la rorippe d'Islande et le pissenlit (Taraxacum officinale Weber) (tableau 2).

Pour l'ensemble des pépinières produisant en pleine terre, la vesce jargeau était l'espèce la plus commune suivie de la rorippe d'Islande, du chiendent, du chénopode, et de l'échinochloa pied-de-coq. À l'exception de la rorippe d'Islande, ces espèces sont généralement communes à d'autres cultures du Québec. Trois des espèces de mauvaises herbes observées fréquemment dans les pépinières, soit la gnaphale des vases (Gnaphalium uliginosum L.), la rorippe d'Islande et la rorippe sylvestre, semblent rares dans d'autres cultures (Doyon et Bouchard 1981; Doyon et al. 1982, 1987a, 1987b, 1987c, 1987d, 1987e; Lemieux et al. 1988a, 1988b, 1988c).

La gnaphale des vases ne semble pas causer de problèmes dans les pépinières. Toutefois, les deux espèces de rorippe sont plus problématiques parce qu'elles persistent malgré les sarclages répétés. La rorippe d'Islande est une plante bisannuelle qui produit une grande quantité de graines (Marie-Victorin 1964) alors que la rorippe sylvestre est une plante vivace qui a une grande capacité de régénération par ses rhizomes (Cloutier et Leblanc 1987a).

Répression des mauvaises herbes dans la production en pleine terre. Les méthodes de lutte utilisées dans la production en pleine terre sont le sarclage manuel, le sarclage mécanique et l'emploi d'herbicides (tableau 3). La maind'oeuvre employée dans ces programmes de répression variait entre les pépinières et n'était pas nécessairement reliée à leur superficie. En moyenne, elle était de 5,6 personnes pendant l'été. Certains pépiniéristes utilisaient rarement le sarclage manuel alors que pour d'autres, c'était leur principale méthode de lutte contre les mauvaises herbes. En général, les pépinières étaient sarclées mécaniquement entre les rangs, de deux à sept fois par année, selon la régie établie par chaque producteur. À l'occasion, certains pépiniéristes effectuaient aussi un sarclage manuel sur le rang.

La fréquence de sarclage était souvent reliée à la taille de la culture ou à la date prévue de vente des plants. Les arbres et arbustes bien établis et de taille supérieure à $1 \mathrm{~m}$ étaient sarclés beaucoup moins souvent que les plantes plus petites ou récemment plantées. L'effort de lutte contre les mauvaises herbes diminuait beaucoup lorsqu'il était prévu que la culture serait vendue au cours de la saison.

Les herbicides étaient surtout utilisés en préplantation lors de la préparation du champ. La moitié des producteurs ne faisaient usage d'herbicides sélectifs que sur une faible proportion de la superficie de leur pépinière (tableau 3). Ceci est attribuable au manque d'information et aux risques de phytotoxicité des herbicides. En effet, les herbicides sélectifs qui peuvent être utilisés en culture ornementale ne sont homologués, en moyenne, que pour 28 espèces ou cultivars (Cloutier et Hamel 1989) alors qu'il y en a plus de 1700 en culture au Québec (Anony- 
Tableau 2. Pourcentage de présence des mauvaises herbes retrouvées dans les pépinières produisant en pleine terre dans diverses régions agricoles du Québec

\begin{tabular}{|c|c|c|c|c|}
\hline \multirow[b]{2}{*}{ Espèce } & \multicolumn{4}{|c|}{ Présence $(\%) \stackrel{\S}{\$}$} \\
\hline & $\begin{array}{c}\text { Région } \\
\text { de Québec } \\
\text { et Mauricie }\end{array}$ & $\begin{array}{c}\text { Région } \\
\text { du Richelieu }\end{array}$ & $\begin{array}{c}\text { Région du } \\
\text { Sud-ouest } \\
\text { et du Nord de } \\
\text { Montréal }\end{array}$ & $\begin{array}{l}\text { Ensemble } \\
\text { du Québec }\end{array}$ \\
\hline Vicia cracia $\mathrm{L}$. & 100 & 80 & 100 & 95 \\
\hline Agropyron repens (L.) Beauv. & 80 & 80 & 100 & 90 \\
\hline Chenopodium spp. & 60 & 100 & 100 & 90 \\
\hline Echinochloa crusgalli (L.) Beauv. & 60 & 100 & 100 & 90 \\
\hline Rorippa islandica (Oeder) Borb. & 80 & 100 & 91 & 90 \\
\hline Amaranthus retroflexus $\mathrm{L}$. & 20 & 100 & 91 & 76 \\
\hline Ambrosia artemisiifolia $\mathrm{L}$. & 40 & 100 & 82 & 76 \\
\hline Polygonum persicaria $\mathrm{L}$. & 20 & 100 & 91 & 76 \\
\hline Taraxacum officinale Weber & 40 & 80 & 91 & 76 \\
\hline Capsella bursa-pastoris (L.) Medic. & 40 & 80 & 82 & 71 \\
\hline Equisetum arvense $\mathrm{L}$. & 60 & 60 & 73 & 67 \\
\hline Panicum capillare $\mathrm{L}$. & 20 & 100 & 73 & 67 \\
\hline Senecio vulgaris $\mathrm{L}$. & 60 & 80 & 64 & 67 \\
\hline Erigeron canadensis $\mathrm{L}$. & 40 & 40 & 82 & 62 \\
\hline Gnaphalium uliginosum L. & 40 & 60 & 73 & 62 \\
\hline Polygonum convolvulus $\mathrm{L}$. & 60 & 40 & 73 & 62 \\
\hline Cyperus esculentus $\mathrm{L}$. & 20 & 60 & 73 & 57 \\
\hline Portulaca oleracea $\mathrm{L}$. & 0 & 80 & 73 & 57 \\
\hline Rorippa sylvestris (L.) Bess. & 60 & 60 & 55 & 57 \\
\hline Stellaria media (L.) Vill. & 20 & 100 & 55 & 57 \\
\hline Asclepias syriaca $\mathrm{L}$. & 20 & 40 & 73 & 52 \\
\hline Chrysanthemum leucanthemum $\mathrm{L}$. & 60 & 40 & 55 & 52 \\
\hline Digitaria sanguinalis (L.) Scop. & 0 & 100 & 55 & 52 \\
\hline Lepidium densiflorum Schrad. & 60 & 40 & 55 & 52 \\
\hline Oxalis stricta $\mathrm{L}$. & 40 & 20 & 73 & 52 \\
\hline Plantago major $\mathrm{L}$. & 20 & 60 & 64 & 52 \\
\hline Setaria glauca (L.) Beauv. & 40 & 40 & 64 & 52 \\
\hline Matricaria matricarioides (Less.) Porter & 80 & 40 & 36 & 48 \\
\hline Rumex acetosella $\mathrm{L}$. & 40 & 20 & 55 & 43 \\
\hline Oenothera biennis L. & 40 & 40 & 27 & 33 \\
\hline Thlaspi arvense $\mathrm{L}$. & 40 & 20 & 36 & 33 \\
\hline Cirsium arvense (L.) Scop. & 20 & 40 & 27 & 29 \\
\hline Erysimum cheiranthoides L. & 20 & 20 & 36 & 29 \\
\hline Rumex crispus $\mathrm{L}$. & 20 & 20 & 36 & 29 \\
\hline Sinapis arvensis $\mathrm{L}$. & 0 & 40 & 36 & 29 \\
\hline Acalypha rhomboidea Raf. & 20 & 20 & 27 & 24 \\
\hline Chenopodium glaucum $\mathrm{L}$. & 0 & 40 & 27 & 24 \\
\hline Daucus carota $\mathrm{L}$. & 20 & 40 & 18 & 24 \\
\hline $\begin{array}{l}\text { Epilobium glandulosum Lehm var. } \\
\text { adenocaulon (Haussk.) Fern. }\end{array}$ & 60 & 20 & 9 & 24 \\
\hline Medicago lupulina L. & 0 & 20 & 36 & 24 \\
\hline Polygonum aviculare $\mathrm{L}$. & 20 & 20 & 27 & 24 \\
\hline Polygonum spp. & 40 & 40 & 9 & 24 \\
\hline
\end{tabular}




\begin{tabular}{|c|c|c|c|c|}
\hline \multirow[b]{2}{*}{ Espèce } & \multicolumn{4}{|c|}{ Présence $(\%)^{\S}$} \\
\hline & $\begin{array}{c}\text { Région } \\
\text { de Québec } \\
\text { et Mauricie }\end{array}$ & $\begin{array}{c}\text { Région } \\
\text { du Richelieu }\end{array}$ & $\begin{array}{c}\text { Région du } \\
\text { Sud-ouest } \\
\text { et du Nord de } \\
\text { Montréal }\end{array}$ & $\begin{array}{l}\text { Ensemble } \\
\text { du Québec }\end{array}$ \\
\hline Potentilla norvegica $\mathrm{L}$. & 40 & 20 & 18 & 24 \\
\hline Solidago canadensis $\mathrm{L}$. & 40 & 20 & 18 & 24 \\
\hline Sonchus asper (L.) Hill & 20 & 20 & 27 & 24 \\
\hline Spergula arvensis $\mathrm{L}$. & 20 & 0 & 36 & 24 \\
\hline Trifolium spp. & 60 & 20 & 9 & 24 \\
\hline Lobelia inflata $\mathrm{L}$. & 60 & 0 & 9 & 19 \\
\hline Polygonum scabrum Moench & 0 & 60 & 9 & 19 \\
\hline Silene vulgaris (Moench) Garcke & 20 & 20 & 18 & 19 \\
\hline Stellaria graminea $\mathrm{L}$. & 20 & 20 & 18 & 19 \\
\hline Achillea millefolium $\mathrm{L}$. & 20 & 0 & 18 & 14 \\
\hline Bidens spp. & 0 & 0 & 27 & 14 \\
\hline $\begin{array}{l}\text { Cerastium fontanum Baumg. subsp. } \\
\text { triviale (Link) Jalas }\end{array}$ & 0 & 20 & 18 & 14 \\
\hline Erigeron philadelphicus $\mathrm{L}$. & 20 & 0 & 18 & 14 \\
\hline Glechoma hederacea $\mathrm{L}$. & 0 & 20 & 18 & 14 \\
\hline Hypericum spp. & 0 & 0 & 27 & 14 \\
\hline Lactuca biennis (Moench) Fern. & 20 & 0 & 18 & 14 \\
\hline Pastinaca sativa $\mathrm{L}$. & 0 & 0 & 27 & 14 \\
\hline Polygonum achoreum Blake & 20 & 0 & 18 & 14 \\
\hline Potentilla argentea $\mathrm{L}$. & 0 & 40 & 9 & 14 \\
\hline Silene alba (Mill.) E.H.L. Krause & 0 & 20 & 18 & 14 \\
\hline Anthemis cotula $\mathrm{L}$. & 20 & 20 & 0 & 10 \\
\hline Artemisia biennis Willd. & 0 & 0 & 18 & 10 \\
\hline Artemisia vulgaris $\mathrm{L}$. & 0 & 0 & 18 & 10 \\
\hline Cirsium vulgare (Savi) Ten. & 20 & 0 & 9 & 10 \\
\hline Erigeron annuus (L.) Pers. & 20 & 0 & 9 & 10 \\
\hline Galeopsis tetrahit $\mathrm{L}$. & 40 & 0 & 0 & 10 \\
\hline Hieracium florentinum All. & 0 & 0 & 18 & 10 \\
\hline Lactuca scariola $\mathrm{L}$. & 0 & 0 & 18 & 10 \\
\hline Linaria canadensis (L.) Dumont & 20 & 0 & 9 & 10 \\
\hline Panicum lanuginosum Ell. & 0 & 0 & 18 & 10 \\
\hline Phleum pratense $\mathrm{L}$. & 0 & 20 & 9 & 10 \\
\hline Phragmites australis (Cav.) Trin. ex Steud. & 0 & 40 & 0 & 10 \\
\hline Pоа аппиа $\mathrm{L}$. & 0 & 0 & 18 & 10 \\
\hline Polygonum pensylvanicum $\mathrm{L}$. & 0 & 40 & 0 & 10 \\
\hline Ranunculus acris L. & 20 & 0 & 9 & 10 \\
\hline Setaria viridis (L.) Beauv. & 0 & 0 & 18 & 10 \\
\hline Sonchus oleraceus L. & 0 & 0 & 18 & 10 \\
\hline Tragopogon pratensis L. & 20 & 0 & 9 & 10 \\
\hline Veronica peregrina $\mathrm{L}$. & 20 & 20 & 0 & 10 \\
\hline Agrostis gigantea Roth & 0 & 0 & 9 & 5 \\
\hline Agrostis scabra Willd. & 0 & 20 & 0 & 5 \\
\hline Amaranthus lividus $\mathrm{L}$. & 0 & 0 & 9 & 5 \\
\hline Apocynum androsaemifolium $\mathrm{L}$. & 0 & 20 & 0 & 5 \\
\hline Arctium minus (Hill) Bernh. & 0 & 0 & 9 & 5 \\
\hline
\end{tabular}


Tableau 2 (suite). Pourcentage de présence des mauvaises herbes retrouvées dans les pépinières produisant en pleine terre dans diverses régions agricoles du Québec

\begin{tabular}{|c|c|c|c|c|}
\hline \multirow[b]{2}{*}{ Espèce } & \multicolumn{4}{|c|}{ Présence $(\%) \stackrel{\S}{\$}$} \\
\hline & $\begin{array}{c}\text { Région } \\
\text { de Québec } \\
\text { et Mauricie }\end{array}$ & $\begin{array}{c}\text { Région } \\
\text { du Richelieu }\end{array}$ & $\begin{array}{l}\text { Région du } \\
\text { Sud-ouest } \\
\text { et du Nord de } \\
\text { Montréal }\end{array}$ & $\begin{array}{l}\text { Ensemble } \\
\text { du Québec }\end{array}$ \\
\hline Aster umbellatus Mill. & 0 & 20 & () & 5 \\
\hline Bidens v'ulgata Greene & 0 & () & 9 & 5 \\
\hline Calamagrostis canadensis (Michx.) Nutt. & 0 & () & 9 & 5 \\
\hline Camelina sativa (L.) Crantz & 20 & () & 0 & 5 \\
\hline Cardamine pensylvanica Mühl & 20 & 0 & 0 & 5 \\
\hline Cichorium intybus L. & 0 & 0 & 9 & 5 \\
\hline Echium vulgare L. & 20 & 0 & 0 & 5 \\
\hline Epilobium coloratum Biehler & 0 & 0 & 9 & 5 \\
\hline Galium mollugo L. & 20 & 0 & 0 & 5 \\
\hline Hordeum jubatum $\mathrm{L}$. & 0 & 0 & 9 & 5 \\
\hline Hypericum majus (A. Gray) Britton & 0 & () & 9 & 5 \\
\hline Juncus spp. & 0 & 0 & 9 & 5 \\
\hline Lactuca spp. & 0 & () & 9 & 5 \\
\hline Linaria vulgaris Mill. & () & 0 & 9 & 5 \\
\hline Lycopus americanus Mühl. & 0 & () & 9 & 5 \\
\hline Lysimachia terrestris (L.) B.S.P. & 20 & 0 & 0 & 5 \\
\hline Malva neglecta Wallr. & 0 & 0 & 9 & 5 \\
\hline Melilotus alba Desr. & 20 & () & 0 & 5 \\
\hline Mentha spp. & 0 & 0 & 9 & 5 \\
\hline Oenothera perennis L. & 0 & () & 9 & 5 \\
\hline Plantago lanceolata L. & 0 & () & 9 & 5 \\
\hline Prunella vulgaris L. & 20 & 0 & () & 5 \\
\hline Ranunculus repens $\mathrm{L}$. & 0 & 20 & 0 & 5 \\
\hline Rumex obtusifolius $\mathrm{L}$. & 20 & 0 & 0 & 5 \\
\hline Rumex persicarioides L. & 0 & 0 & 9 & 5 \\
\hline Rumex verticillatus L. & 0 & () & 9 & 5 \\
\hline Scleranthus annutus L. & 0 & 0 & 9 & 5 \\
\hline Sisymbrium altissimum L. & 0 & 20 & 0 & 5 \\
\hline Sonchus arvensis L. & 0 & 0 & 9 & 5 \\
\hline Trifolium pratense $\mathrm{L}$. & 0 & 20 & () & 5 \\
\hline Tussilago farfara $\mathrm{L}$. & 20 & () & () & 5 \\
\hline Viola arvensis Murr. & 0 & 20 & 0 & 5 \\
\hline Viola tricolor $\mathrm{L}$. & 0 & 0 & 9 & 5 \\
\hline Xanthium strumarium L. & 0 & 0 & 9 & 5 \\
\hline
\end{tabular}

$\$$ Rapport en pourcentage du nombre de pépinières où la mauvaise herbe était présente sur le nombre total de pépinières visitées.

me 1986). Par conséquent, l'innocuité des herbicides est inconnue pour $98 \%$ des espèces ou cultivars. Un seul herbicide peut être utilisé pratiquement partout mais il n'est efficace que contre un faible nombre d'espèces de mauvaises herbes. L'échelle restreinte de production de chaque espèce à l'intérieur d'une pépinière résulte en une mosaïque d'espèces ou de variétés de plantes ornementales sur la même aire de production. Celles-ci réagissent souvent de manières différentes aux herbicides et compliquent ainsi leur utilisation dans les pépinières. 
Tableau 3. Méthodes de répression utilisées en production ornementale au Québec

\begin{tabular}{|c|c|c|}
\hline \multirow[b]{2}{*}{ Méthode de répression } & \multicolumn{2}{|c|}{$\begin{array}{l}\text { Fréquence d'utilisation } \\
\text { (\% des pépinières) }\end{array}$} \\
\hline & $\begin{array}{c}\text { Production } \\
\text { en pleine } \\
\text { terre }\end{array}$ & $\begin{array}{c}\text { Production } \\
\text { en } \\
\text { contenants }\end{array}$ \\
\hline \multicolumn{3}{|l|}{ Chimiques } \\
\hline $2,4-\mathrm{D}$ & 14 & 0 \\
\hline 2,4-D/mécoprop/dicamba & 5 & 0 \\
\hline chlorthal-diméthyl & 5 & 0 \\
\hline dichlobénil & 10 & 0 \\
\hline difénamide & 14 & 18 \\
\hline fluazifop-butyl & 14 & 9 \\
\hline glyphosate & 76 & 0 \\
\hline paraquat & 43 & 9 \\
\hline simazine & 33 & 36 \\
\hline trifluraline & 5 & 9 \\
\hline chloroxuron & 0 & 9 \\
\hline napropamide & 0 & 9 \\
\hline oxadiazon & 0 & 9 \\
\hline Mécaniques & 100 & - \\
\hline Manuelles & 100 & 100 \\
\hline \multicolumn{3}{|l|}{ Prélentives } \\
\hline terreau acheté & - & 36 \\
\hline terreau fait sur place & - & 64 \\
\hline paillis de plastique sur le sol & - & 55 \\
\hline couvre-pot & - & 27 \\
\hline
\end{tabular}

Cependant, deux herbicides non-sélectifsétaient employés fréquemment: le paraquat (ion de 1,1'-diméthyl-4,4'-bipyridinium) et le glyphosate ( $N$-[phosphonométhyl]glycine). La nomenclature des herbicides suit celle de la Weed Science Society of America (Anonyme 1990). Ces produits étaient généralement appliqués localement sur les populations de mauvaises herbes visées.

Dans la production en pleine terre, la présence et la densité des mauvaises herbes annuelles et vivaces étaient influencées par la régie de production. Malgré le faible niveau d'infestation des diverses espèces, les tendances suivantes ont été observées. 1) Les mauvaises herbes vivaces étaient pratiquement les seules à être observées dans les champs lorsque la fréquence de sarclage était élevée. Cette pratique culturale est très efficace contre les annuelles mais permet ainsi aux vivaces de croître sans compétition.
De plus, le sarclage multiplie les vivaces en sectionnant leurs parties végétatives. 2) Les infestations de stellaire moyenne et de pourpier potager pouvaient devenir très importantes en août et en septembre lorsque le sarclage cessait tôt en saison. Ce phénomène peut s'expliquer par la germination tardive de ces deux espèces (Miyanishi et Cavers 1980; Turkington et al. 1980). De plus, certaines autres espèces de mauvaises herbes peuvent s'implanter en août et en septembre, et continuer leur cycle vital l'été suivant. Le printemps est une période de très grande activité dans les pépinières et le premier sarclage de l'année tend à être tardif. Les mauvaises herbes de l'année précédente ont donc le temps de bien s'établir, ce qui les rend difficiles à éliminer. 3) Les mauvaises herbes vivaces s'établissaient sur les rangs et pouvaient parfois dépasser la taille des cultures lorsque le sarclage n'était effectué qu'entre les rangs. 
Tableau 4. Pourcentage de présence des mauvaises herbes inventoriées dans les 12 pépinières produisant en contenants

\begin{tabular}{|c|c|c|c|}
\hline Espèce & Présence $(\%)$ & Espèce & Présence $(\%)^{\$}$ \\
\hline Senecio vulgaris $\mathrm{L}$. & 67 & Matricaria matricarioides (Less.) Porter & 17 \\
\hline Chenopodium spp. & 58 & Polygonum convolvulus $\mathrm{L}$. & 17 \\
\hline Echinochloa crusgalli (L.) Beauv. & 58 & Ranunculus acris L. & 17 \\
\hline Stellaria media (L.) Vill. & 58 & Rorippa sylvestris (L.) Bess. & 17 \\
\hline Capsella bursa-pastoris (L.) Medic. & 50 & Setaria glauca (L.) Beauv. & 17 \\
\hline Cyperaceae & 50 & Tussilago farfara $\mathrm{L}$. & 17 \\
\hline Polygonum persicaria $\mathrm{L}$. & 50 & Asclepias syriaca $\mathrm{L}$. & 8 \\
\hline Vicia cracca $\mathrm{L}$. & 50 & Bidens spp. & 8 \\
\hline Oxalis stricta $\mathrm{L}$ & 42 & Carex spp. & 8 \\
\hline Panicum capillare L. & 42 & Cerastium spp. & 8 \\
\hline Portulaca oleracea $\mathrm{L}$. & 42 & Chenopodium glaucum $\mathrm{L}$. & 8 \\
\hline Rorippa islandica (Oeder) Borb. & 42 & Daucus carota $\mathrm{L}$. & 8 \\
\hline Taraxacum officinale Weber & 42 & Digitaria sanguinalis (L.) Scop. & 8 \\
\hline Agropyron repens (L.) Beauv. & 33 & Epilobium coloratum Biehler & 8 \\
\hline Cardamine pensylvanica Mühl & 33 & Euphorbia helioscopia L. & 8 \\
\hline Equisetum arvense $\mathrm{L}$. & 33 & Galinsoga quadriradiata Ruiz \& Pav. & 8 \\
\hline Gnaphalium uliginosum $\mathrm{L}$. & 33 & Juncus spp. & 8 \\
\hline Plantago major L. & 33 & Phleum pratense L. & 8 \\
\hline Po a spp. & 33 & Rumex acetosella L. & 8 \\
\hline $\begin{array}{l}\text { Ambrosia artemisiifolia } \mathrm{L} \text {. } \\
\text { Epilobium glandulosum } \mathrm{Lehm} \text { var. }\end{array}$ & $\begin{array}{l}25 \\
25\end{array}$ & Sinapis arvensis L. & 8 \\
\hline adenocaulon (Haussk.) Fern. & $2 J$ & Thlaspi arvense $\mathrm{L}$. & 8 \\
\hline Erysimum cheiranthoides L. & 25 & Trifolium agrarium $\mathrm{L}$. & 8 \\
\hline Medicago lupulina $\mathrm{L}$. & 25 & Trifolium spp. & 8 \\
\hline Solidago canadensis L. & 25 & Urtica dioica $\mathrm{L}$. & 8 \\
\hline Erigeron canadensis L. & 17 & Veronica peregrina $\mathrm{L}$. & 8 \\
\hline
\end{tabular}

$\S$ Rapport en pourcentage du nombre de pépinières où la mauvaise herbe était présente sur le nombre total de pépinières visitées.

Inventaire des mauvaises herbes dans la production en contenants. Parmi les pépinières visitées au cours de l'été, 12 pépiniéristes produisaienten contenants. Bienqu'ellesétaient réparties dans toutes les régions, ces pépinières étaient particulièrement concentrées dans la région de Québec (tableau 1). La liste des mauvaises herbes inventoriées dans les contenants de toutes les pépinières est présentée au tableau 4. Parmi ces espèces, le séneçon vulgaire (Senecio vulgaris L.) était rencontré le plus fréquemment, suivi par le chénopode, l'échinochloa pied-de-coq et la stellaire moyenne.

Les mauvaises herbes observées dans les contenants étaient des espèces communes aux autres productions québécoises, à l'exception de la rorippe d'Islande, de la cardamine de Pennsylvanie (Cardamine pensylvanica Mühl) et de l'oxalide d'Europe (Oxalis stricta L.)
(Doyon et Bouchard 1981; Doyon et al. 1982, 1987a, 1987b, 1987c, 1987d, 1987e; Lemieux et al. 1988a, 1988b, 1988c). La cardamine de Pennsylvanie est une plante annuelle, la rorippe d'Islande, une bisannuelle et l'oxalide d'Europe, une vivace (Marie-Victorin 1964). Des infestations importantes de cardamine de Pennsylvanie ont été observées surtout dans la région de Québec. Cette espèce semble posséder un fort potentiel d'envahissement parce qu'elle peut compléter plus d'un cycle vital par année et qu'elle peut projeter ses graines à plus de $1 \mathrm{~m}$ de distance (Cloutier et Leblanc 1987b).

La contamination des contenants semble être causée par le mode de dispersion des graines: soit par le vent (séneçon vulgaire), par projection lors de l'ouverture du fruit (cardamine de Pennsylvanie et oxalide d'Europe) ou par gravité lorsque les graines tombent directement 
Tableau 5. Nombre et pourcentage de présence des mauvaises herbes présentes dans le terreau utilisé pour la production en contenants $\$$

\begin{tabular}{|c|c|c|}
\hline Espèce & $\begin{array}{l}\text { Nombre total } \\
\text { de plantules }\end{array}$ & Présence $(\%)^{\dagger}$ \\
\hline Cardamine pensylvanica Mühl & 39 & 86 \\
\hline Poa annua L. & 35 & 86 \\
\hline Cyperaceae & 4 & 57 \\
\hline Chenopodium spp. & 4 & 43 \\
\hline Plantago major $\mathrm{L}$. & 4 & 43 \\
\hline Potentilla norvegica $\mathrm{L}$. & 6 & 43 \\
\hline Veronica peregrina $\mathrm{L}$. & 7 & 43 \\
\hline Alopecurus aequalis Sobol. & 5 & 29 \\
\hline Capsella bursa-pastoris (L.) Medic. & 5 & 29 \\
\hline Chenopodium glaucum $\mathrm{L}$. & 6 & 29 \\
\hline Erigeron spp. & 2 & 29 \\
\hline Juncus bufonius L. & 8 & 29 \\
\hline Juncus tenuis Willd. & 8 & 29 \\
\hline Oxalis stricta $\mathrm{L}$. & 11 & 29 \\
\hline Sonchus asper (L.) Hill & 2 & 29 \\
\hline Taraxacum officinale Weber & 4 & 29 \\
\hline Trifolium pratense $\mathrm{L}$. & 2 & 29 \\
\hline Amaranthus retroflexus $\mathrm{L}$. & 1 & 14 \\
\hline Ambrosia artemisiifolia $\mathrm{L}$. & 1 & 14 \\
\hline Artemisia absinthium $\mathrm{L}$. & 1 & 14 \\
\hline Carex spp. & 1 & 14 \\
\hline Cerastium fontanum Baumg. subsp. triviale (Link) Jalas & 1 & 14 \\
\hline Chenopodium album $\mathrm{L}$. & 1 & 14 \\
\hline Digitaria sanguinalis (L.) Scop. & 1 & 14 \\
\hline Echinochloa crusgalli (L.) Beauv. & 1 & 14 \\
\hline Epilobium glandulosum Lehm var. adenocaulon (Haussk.) Fern. & 1 & 14 \\
\hline Erigeron canadensis L. & 1 & 14 \\
\hline Erysimum cheiranthoides $\mathrm{L}$. & 1 & 14 \\
\hline Galinsoga quadriradiata Ruiz \& Pav. & 1 & 14 \\
\hline Hypericum ellipticum Hook. & 4 & 14 \\
\hline Hypericum mutilum $\mathrm{L}$. & 1 & 14 \\
\hline Hypericum perforatum $\mathrm{L}$. & 1 & 14 \\
\hline Hypericum spp. & 27 & 14 \\
\hline Oenothera biennis $\mathrm{L}$. & 1 & 14 \\
\hline Poa palustris L. & 1 & 14 \\
\hline Polygonum scabrum Moench & 3 & 14 \\
\hline Portulaca oleracea $\mathrm{L}$. & 3 & 14 \\
\hline Potentilla spp. & 1 & 14 \\
\hline Rorippa islandica (Oeder) Borb. & 2 & 14 \\
\hline Rumex spp. & 10 & 14 \\
\hline Senecio vulgaris $\mathrm{L}$. & 1 & 14 \\
\hline Sonchus spp. & 1 & 14 \\
\hline Stellaria media (L.) Vill. & 1 & 14 \\
\hline Trifolium hybridum L. & 1 & 14 \\
\hline Trifolium spp. & 1 & 14 \\
\hline Urtica dioica $\mathrm{L}$. & 8 & 14 \\
\hline Vicia cracca $\mathrm{L}$. & 1 & 14 \\
\hline
\end{tabular}

$\S$ Somme des espèces dénombrées dans le terreau provenant de sept pépinières. Le volume total de terreau est $0,105 \mathrm{~m}^{3}$.

$\doteqdot$ Rapport en pourcentage du nombre de pépinières où la mauvaise herbe était présente sur le nombre total de pépinières visitées. 
sur le dessus du pot (le chénopode, l'échinochloa pied-de-coq et la stellaire moyenne) (Whitcomb 1984). L'eau d'irrigation peut aussi devenir une source de contamination si des graines de mauvaises herbes atteignent le bassin (Whitcomb 1984).

Contamination du terreau. Le substrat utilisé variait considérablement car 64\% des entreprises faisaient leur propre mélange. En général, il était composé de sable, de tourbe de sphaigne, de bran de scie, de terre noire ou de compost. Les autres achetaient du terreau préparé et pré-mélangé. Les échantillons de terreau contenaient tous des graines de mauvaises herbes, bien que la majorité des échantillons étaient considérés comme stériles par les producteurs (tableau 5). En moyenne, 6,7 espèces de mauvaises herbes ont été identifiées et 33,1 plantes par volume de $0,015 \mathrm{~m}^{3}$ de terreau ont été dénombrées au cours de cette expérience. Ce volume de sol est équivalent à quatre contenants de $4 \mathrm{~L}$ utilisés habituellement dans les pépinières.

La cardamine de Pennsylvanie, le pâturin annuel (Poa annua L.) et les cypéracées étaient les mauvaises herbes dominantes dans les terreaux. La disparité observée entre les espèces de mauvaises herbes infestant les contenants et celles contaminant le terreau avant son utilisation (tableau 4 et 5) dépend entre autres de la dormance des graines de mauvaises herbes, de la contamination du contenant une fois celui-ci placé sur l'aire de production et de la période écoulée depuis le dernier sarclage à la prise de données en champs.

Répression des mauvaises herbes dans les contenants. Les méthodes de lutte utilisées contre les mauvaises herbes dans la production en contenants étaient la prévention, le sarclage manuel et l'emploi d'herbicides.

Une première méthode préventive consistait à faire usage de terreau stérile (36\% des pépinières) et à s'assurer qu'il reste exempt de graines de mauvaises herbes avant son utilisation. S'il était entreposé à l'extérieur, certains producteurs le recouvraient d'une toile et détruisaient toutes les mauvaises herbes qui étaient à proximité. Une fois placés en aire de production, les contenants peuvent rester exempts de mauvaises herbes pendant 1 ou 2 mois (observation personnelle). L'utilisation de couvre-pots était une méthode préventive utilisée par $27 \%$ des pépinières. Ces dispositifs prévenaient l'établissement d'une partie des mauvaises herbes qui, autrement, pourraient s'implanter dans le contenant. Une autre méthode de lutte préventive consistait à détruire les mauvaises herbes autour du bassin d'irrigation car de nombreuses graines entraînées par l'eau d'irrigation peuvent contaminer les pots. L'utilisation de pellicule de plastique opaque sur le sol où sont placés les contenants était une autre méthode préventive. Celle-ci était utilisée par 55\% des producteurs. Elle empêche la croissance de la majorité des mauvaises herbes présentes dans le sol, prévenant ainsi la contamination des pots par les graines qu'elles produiraient éventuellement.

Le désherbage manuel, seul ou combiné avec d'autres méthodes de répression, était pratiqué dans toutes les pépinières. En général, il était effectué au moins deux fois au cours de la saison de croissance. Cette pratique est dispendieuse et peut nuire à la culture si trop de terreau est enlevé avec la mauvaise herbe (Whitcomb 1984).

Huit herbicides étaient utilisés sur les contenants dans les pépinières (tableau 3 ) mais un seul est homologué au Canada (Cloutier et Hamel 1989). Celui-ci ne peut être utilisé que sur 14 espèces ornementales. L'utilisation d'herbicides n'était pratiquée que par 36\% des pépiniéristes dans la production en contenants pour les raisons énoncées pour la production en pleine terre.

En résumé, les infestations de mauvaises herbes étaient maintenues à de faibles niveaux dans les pépinières visitées grâce à une lutte dirigée continue. Cependant, selon les pépiniéristes, le désherbage demeure une des opérations les plus coûteuses dans les pépinières. Certaines espèces de mauvaises herbes telles que la vesce jargeau, le chiendent, la prèle des champs, le souchet comestible, la rorippe d'Islande et la rorippe sylvestre pourraient devenir prédominantes si la fréquence des sarclages diminuait à la suite d'une plus grande utilisation d'herbicides commeilaété observépourd'autres mauvaises herbes dans des cultures sarclées telles le maïs ou le soya (Buchanan et al. 1975; Doyon et Bouchard 1981). Le séneçon vulgaire était l'espèce la plus fréquente dans la production en contenants et la cardamine de Pennsylvanie est une espèce à surveiller à cause de son fort potentiel d'envahissement. 
Les auteurs désirent remercier les producteurs qui ont accepté de participer à l'inventaire et à l'enquête.

Alex, F. J., R. Cayouette et G. Mulligan. 1980. Noms populaires et scientifiques des plantes nuisibles au Canada. Direction de la recherche, Agriculture Canada, Ottawa, Canada. Publication 1397. $132 \mathrm{pp}$.

Anonyme. 1986. Guide des disponibilités 1986-87 des plants de pépinière. Québec Vert, Vol.8, Sept. 1986, Annexe. $64 \mathrm{pp}$.

Anonyme. 1990. Common and chemical names of herbicides approved by the Weed Science Society of America. Weed Sci. 38: 639-646.

Buchanan, G.A., C.S. Hoveland, V.L. Brown et R.H. Wade. 1975. Weed population shifts influenced by crop rotations and weed control programs. Proc. South. Weed Sci. Soc. 28: 60-71.

Cloutier, D. et J.M. Hamel. 1989. Pépinières ornementales, répression des mauvaises herbes. Conseil des productions végétales du Québec, ministère de l'Agriculture, des Pêcheries et de l'Alimentation du Québec. Agdex 270-640. 46 pp.

Cloutier, D. et M. Leblanc. 1987a. Capacité de régénération des rhizomes de rorippe sylvestre. Rap. Rech., Comité d'experts en malherbologie (Est du Canada) 33: 619 .

Cloutier, D. et M. Leblanc. 1987b. Dispersion des graines de cardamine de Pennsylvanie. Rap. Rech., Comité d'experts en malherbologie (Est du Canada) 33: 619 .

Doyon, D. et C.J. Bouchard. 1981. Inventaires des mauvaises herbes dans les champs de maïs-grain du comté de Saint-Hyacinthe, Québec. Phytoprotection 62: $1-10$.

Doyon, D., J.-M. Deschênes, C.-J. Bouchard et R. Rioux. 1982. Les inventaires de mauvaises herbes dans les principales cultures au Québec. I. Buts et méthodologie. Phytoprotection 63: 10-21.

Doyon, D., C.J. Bouchard et R. Néron. 1987a. Inventaire des mauvaises herbes dans les cultures du Québec (1980-1984). Vol. 1: Région agricole de Québec (02). Mém. Serv. Rech. Phytotech. Qué. N4, Ministère de l'Agriculture, des Pêcheries et de l'Alimentation du Québec. 41 pp.

Doyon, D., C.J. Bouchard et R. Néron. 1987b. Inventaire des mauvaises herbes dans lescultures du Québec (1980-1984). Vol. 2: Région agricole des Cantonsde-l'Est (05). Mém. Serv. Rech. Phytotech. Qué. Nº4, Ministère de l'Agriculture, des Pêcheries et de l'Alimentation du Québec. 49 pp.

Doyon, D., C.J. Bouchard et R. Néron. 1987c. Inventaire des mauvaises herbes dans les cultures du Québec (1980-1984). Vol. 3: Région agricole du Richelieu (06). Mém. Serv. Rech. Phytotech. Qué. No 4, Ministère de l'Agriculture, des Pêcheries et de l'Alimentation du Québec. 93 pp.
Doyon, D., C.J. Bouchard et R. Néron. 1987d. Inventaire des mauvaises herbes dans les cultures du Québec (1980-1984). Vol. 4: Région agricole du Sud-Ouest de Montréal (07). Mém. Serv. Rech. Phytotech. Qué. $\mathrm{N}^{\circ} 4$, Ministère de l'Agriculture, des Pêcheries et de l'Alimentation du Québec. 102 pp.

Doyon, D., C.J.Bouchard et R. Néron. 1987e. Inventaire des mauvaises herbes dans les cultures du Québec (1980-1984). Vol. 5: Région agricole de L'Assomption (10). Mém. Serv. Rech. Phytotech. Qué. No 4 , Ministère de l'Agriculture, des Pêcheries et de l'Alimentation du Québec. $131 \mathrm{pp}$.

Lemieux, C., A. Larouche, A. Légère, J.-M. Deschênes et R. Rioux. 1988a. Inventaire des mauvaises herbes au Québec. Céréales pures et plantes-abri. Bull. Ext. No 2. Sta. Rech. Sainte-Foy, Agric. Can. 84 pp.

Lemieux, C., A. Larouche, A. Légère, J.-M. Deschênes et R. Rioux. 1988b. Inventaire des mauvaises herbes au Québec. Maïs, pommes de terre, fraises. Bull. Ext. $N^{\circ} 3$. Sta. Rech. Sainte-Foy, Agric. Can. 45 pp.

Lemieux, C., A. Larouche, A. Légère, J.-M. Deschênes et R. Rioux. 1988c. Inventaire desmauvaises herbes au Québec. Prairies en implantation, prairies établies, pâturages. Bull. Ext. N ${ }^{\circ}$ 4. Sta. Rech. Sainte-Foy, Agric. Can. 64 pp.

MAPAQ (Ministère de l'Agriculture, des Pêcheries et de I'Alimentation du Québec). 1985. Pépinières du Québec inspectées et approuvées en 1984. Gouv. Qué., Min.Agric. Pêch. Alim. Qué., Serv. Rech. Déf. Cult. 46 pp.

MAPAQ (Ministère de l'Agriculture, des Pêcheries et de l'Álimentation du Québec). 1987. Pépinières du Québec inspectées et approuvées en 1986. Gouv. Qué., Min.Agric. Pêch. Alim. Qué., Serv. Prod. Vég. $52 \mathrm{pp}$.

Marie-Victorin, Frère. 1964. Flore Laurentienne, $2^{\mathrm{e}}$ éd. Presses Univ. Montréal. 927 pp.

Miyanishi, K. et P.B. Cavers. 1980. The biology of canadian weeds. 40. Portulaca oleracea L. Can. J. Plant Sci. 60: 953-963.

Robinson, D.W. 1983. Weed control in nursery stock and amenity plantings. Pages 199-226 in: W.W. Fletcher (ed.), Recent advances in weed research. Commonw. Agric. Bur., Farnham Royal, Slough, England. 266 pp.

Scoggan, H. J. 1978-1979. The flora of Canada. Vol. 1 à 4. Publ. Bot. Nat. Museum Nat.Sci. (Canada) No 7 . $1711 \mathrm{pp}$.

Statistique Canada. 1990. Enquête sur l'industrie des pépinières canadiennes, 1988-1989. Statistique $\mathrm{Ca}-$ nada, Division de l'agriculture, Unité de l'horticulture. Min. Approv. Serv., Can. Publ. 22-203. 20 pp.

Turkington, R., N.C. Kenkel et G.D. Framko. 1980. The biology of canadian weeds. 42. Stellaria media (L.) Vill. Can. J. Plant Sci. 60: 981-992.

Whitcomb, C.E. 1984. Plant production in containers. Lacebark Publications, Stillwater, Oklahoma. p. 495557. 